\title{
Ist das Wohl des Landesherrn in Wein zu trinken?
}

Carl und Gerhart Hauptmann bei August Forel in Zürich

Von Hans H. Walser

Gerhart Hauptmann (1862-1946) verbrachte im Jahre 1888 kurze, aber schicksalhaft entscheidende Monate bei seinem Bruder Carl in Zürich. Nach seinen eigenen Worten war diese Zeit für ihn «epochemachend» wegen ihrer «Fülle neuer Erscheinungen»; er traf hier auf «Persönlichkeiten verschiedenster Art» und auf «soziale Institutionen, Parteigebilde kämpferischpolitischer Art», die ihn tief prägten. Zu den großen Eindrücken zählten die Persönlichkeiten von August Forel und die Besuche seiner Vorlesungen und seiner psychiatrischen Klinik. «Er ist es, dessen Erschließungen von überwiegendem Einfluß auf mich gewesen sind. Er hat mir ein unverlierbares Kapital von Wissen um die menschliche Psyche vermittelt», schrieb Gerhart Hauptmann später ${ }^{1}$. Der Zürcher Aufenthalt war aber auch für seine psychische Gesundheit wichtig ${ }^{2}$. Spuren dieser Zürcher Zeit finden sich auch in Gerhart Hauptmanns dramatischen Werken.

Carl Hauptmann (1858-1921) hatte in Jena Naturwissenschaften studiert und 1883 promoviert ${ }^{3}$. 1885 war er für einige Jahre nach Zürich gegangen, wo er sich besonders an Richard Avenarius anschloß. Er hatte die Absicht, sich in Philosophie zu habilitieren; eine Zeitlang dachte er auch daran, Psychiater, also Arzt, zu werden. In Zürich erfolgte, wie Anna Stroka $^{4}$ nachgewiesen hat, die Wandlung Carl Hauptmanns vom philosophisch orientierten Naturwissenschafter zum Dichter ${ }^{5}$. Das Heim von Carl und Martha Hauptmann ${ }^{6}$ war damals nach den Worten von Gerhart Hauptmann «etwas wie eine Platonische Akademie, die allerdings mehr den Geist des 〈Gartens〉, will heißen des Epikureischen Kreises atmete». Namen wie Alfred Ploetz, Ferdinand Simon, Frank Wedekind, Karl Henckell, Pauline Rüdin, Josepha Kodis, Justus Gaule werden genannt. Das damalige Zürich war die Stadt von Gottfried Keller, Arnold Böcklin und Conrad Ferdinand Meyer, die Stadt der werdenden Sozialdemokratie, des Frauenstudiums, der polnischen und russischen Studenten - aber auch die Stadt solider Bürgerlichkeit.

August Forel (1848-1931) muß wohl nicht besonders vorgestellt werden ${ }^{7}$. Als Schüler von Bernhard Gudden und von Theodor Meynert war er 
1879 als sehr junger Mann zum Direktor der psychiatrischen Universitätsklinik («Irrenanstalt Burghölzli») ernannt worden. Als die Brüder Hauptmann ihn kennenlernten, war er etwa vierzigjährig, ebenso sprühend lebendig wie charakterfest - ein «Feuergeist», der die akademische Jugend anzog und für seine Ideale begeisterte. Gerade damals wechselten seine wissenschaftlichen Interessen von der experimentellen Hirnforschung zum vertieften Studium der Psyche mittels des Hypnotismus, und zur Prophylaxe der wichtigsten Suchtkrankheit, des Alkoholismus, durch Abstinenz. Beides beeinflußte die Brüder Hauptmann tief. Besonders der Kampf gegen den Alkohol wurde für Forel ein Teil seines «ärztlichen Apostolates». Carl Hauptmann wurde durch ihn zum überzeugten Abstinenten.

Man wird sich fragen müssen, wie die Brüder Hauptmann so schnell in gesellschaftlichen und wissenschaftlichen Kontakt mit August Forel kommen konnten. Forel hielt sich nämlich vom konventionellen gesellschaftlichen Leben fern und konnte Unbefugte recht schroff aus seinen Vorlesungen weisen. Es ist anzunehmen, daß beide durch den Historiker Arthur Böhtlingk (1849-1929) an Forel empfohlen worden waren. Böhtlingk war ein alter Bekannter der Familie Forel ${ }^{8}$; die Hauptmanns kannten ihn von der Universität Jena her, wo er Gerhart Hauptmanns Mentor gewesen war ${ }^{9}$.

Ein beträchtlicher Teil des Briefwechsels von August Forel befindet sich in Zürich, der wichtigsten Stätte seines akademischen Wirkens. Eine Auswahl aus dieser Korrespondenz ist publiziert ${ }^{10}$. In der Briefsammlung fehlen leider die Antwortbriefe von Forels Hand meistens. Es war deshalb ein Glücksfall, als wir durch Vermittlung von Frau Anna Stroka in Wroclaw (Polen) Kopien von fünf Briefen erhalten konnten, die August Forel an Carl Hauptmann geschrieben hat ${ }^{11,12}$. Das wichtigste Problem, das sich darin findet, betrifft die Alkoholabstinenz, insbesondere die Frage eines eventuellen Dispenses vom feierlichen Abstinenzgelübde.

Der uns bisher bekannte Briefwechsel zwischen den beiden Männern umfaßt elf Briefe von Carl Hauptmann aus der Zeit vom 5. Juni 1886 bis zum 9. Dezember 1890 und fünf Briefe von August Forel aus dem ersten Halbjahr 1888 (1. Januar bis 6. Juli 1888). Die wichtigsten dieser Schriftstücke sollen hier wiedergegeben werden. Bereits publizierte Briefe werden, des Zusammenhanges wegen, kurz resümiert.

Im Juni und Juli 1888 befand sich Carl Hauptmann im Militärdienst im Königreich Sachsen. Am 26. Juni 1888 schrieb er an Forel, seine Abstinenz errege unter den Offizieren «teilweise Ärgernis». Das lasse ihn zwar gleichgültig; doch dränge ihn sein Vorgesetzter, ein Dispensgesuch bezüglich 
seiner Alkoholabstinenz einzuholen. Als Untergebener müsse er diesem Drängen Folge leisten, doch bitte er Forel, das Dispensgesuch zurückzuweisen, jedoch «hinzuzufügen, daß, wenn es militärische Rücksichten dringend erheischen sollten, ... das Wohl des Landesherrn oder des sonst Gefeierten in Wein, und nicht, was ebenso gut ginge, in Wasser zu trinken, ... einige Schluck pro forma ausnahmsweise gestattet werden könnten». ${ }^{13}$

August Forel kam dieser Bitte unverzüglich nach und schrieb drei Tage später:

\section{August Forel an Carl Hauptmann, 29. Juni 1888}

Die Direction der

Kantonalen Heilanstalt

Burghölzli, den 29 VI 1888

Burghölzli Zürich

\section{Hochgeehrter Herr Doctor!}

Sie ersuchen mich um ein Dispens Ihres Abstinenzgelübdes für militärische Festlichkeiten. Ich erlaube mir durch Zusendung eines Kartenformulares (desselben, das Sie unterschrieben haben) in Erinnerung zu bringen, dass eine solche Dispensation absolut unzulässig ist, denn im Formular sind die einzigen zulässigen Ausnahmen (ärztliche Vorschrift und Abendmahlgenuss) ausdrücklich erwähnt. Dass aber diese Unzulässigkeit nicht nur eine formelle, sondern eine sachliche ist, geht daraus hervor, dass der ganze Zweck der Abstinenzbewegung die wir vertreten dahin geht:

1) dem allgemein getriebenen Missbrauch der alcoholischen Getränke, der den Menschen sittlich und körperlich herabwürdigt und schwer schädigt entgegenzutreten -

2) dem eingewurzelten Vorurtheil als ob der Alkoholgenuss den Menschen stärke, und als ob geselliges Zusammensein und Festlichkeiten nicht so gut, und so herzlich ohne Alkohol als mit Alkohol stattfinden könnten durch die Tat entgegenzuarbeiten, dieses Vorurtheil durch Leistung des Gegenbeweises allmälig zu vernichten.

Es ist nun sonnenklar, dass Ihr Dispensgesuch Ihrem gegebenen Ehrenwort nicht nur formell, sondern auch sachlich widerspricht - und ich kenne Ihren Charakter genug um überzeugt zu sein, dass nur der Druck äusserer Verhältnisse (wohl militärischer Natur) Sie zur Einreichung desselben haben veranlassen können. So gerne ich Ihnen persönlich beistehen würde, so sehen Sie nun wohl ein, dass ich es in diesem Falle unmöglich tun kann.

Sollten gewisse militärische Rücksichten den Charakter der «höheren Gewalt» an sich tragen; sollte z. B. bei einem officiellen Feste die militärische Disciplin absolut erfordern, dass das Wohl des Landesherrn oder des sonst Gefeierten in Wein getrunken werde, so könnte es nachträglich entschuldigt werden, wenn bei dieser Gelegenheit der officielle Schluck in Wein getrunken worden ist - nicht aber in der eigentlichen Form eines Dispenses, sondern weil wir uns schliesslich den Landesgesetzen unterordnen müssen nolens volens. - Für diesen Fall sind 
Sie absolviert, aber nur für diesen Fall, und obwohl nach unserer Ansicht der Schluck Wasser, den der Abstinent auf das Wohl seines Landesherren, oder seines Vaterlandes trinkt, an Treue, Aufrichtigkeit und innerem Werth sich mit dem Weinschluck eines jeden Anderen zum mindesten als ebenbürtig messen kann.

Gerade heute sind es zwei Jahre, dass ich mein Abstinenzgelübde gegeben habe. In diesen zwei Jahren habe ich so viel gearbeitet wie nie vorher, war bei allen Festlichkeiten stets sehr fidel und habe nicht nur sehr oft mit Wasser angestossen sondern selbst eine grosse Reihe Wassertoaste vorgebracht. Das alles ging ohne Schwierigkeiten vor sich und hat gut gewirkt.

Herzliche Grüsse von meiner Familie und mir selbst Ihr hochachtungsvollst ergebener Professor Dr. Aug. Forel

So weit der «offizielle» Brief, den Carl Hauptmann seinem Vorgesetzten vorlegen konnte. Aber diesem Schreiben ließ Forel gleich am folgenden Tag einen rein persönlichen Brief folgen:

August Forel an Carl Hauptmann, 30. Juni 1888

Lieber Freund!

Burghölzli 30. VI. 88

Hoffentlich wird Ihnen mein officieller Brief von gestern richtig gedient haben.

$O$ deutscher Zopf

O deutscher Zopf

Wie lang wirst du bestehen

Hast du vor, dich im eignen Kopf

Chinesisch auszudehnen

Pardon! Ich werde Dichter, was sonst nicht mein Beruf ist. Aber doch gebe ich Ihnen die Erlaubnis diesen Vers Ihrem Hauptmann mit einem schönen Gruss von mir vorzutragen, da der tote Chamisso es nicht mehr tun kann.

Hoffentlich wird Ihre Prüfung bald aus sein. Gestern waren meine zwei Jahre aus. Von heute an trinke ich wieder Experimenti Causa Wein für einen Monat. Dann gilt die Abstinenz lebenslänglich, und geselle ich mich dem Freund Ploetz.

Denken Sie: Ein junger stud. juris - Sohn von Advocat Goll hat sich für ein Jahr unterschrieben und zeigt grossen Eifer. Er will sich der Sache recht annehmen und ist der Idee der Gründung eines freisinnigen Abstinenzvereins nicht abgeneigt.

Heute und morgen habe ich Besuch des Präsidenten des holländischen Mässigkeitsvereins, des Herrn Pfarrer Koch, der mir recht gut gefällt.

Mein Vortrag über die Behandlung des Alkoholismus, den ich in Freiburg in Baden kürzlich hielt, ist in der Münchener Medicinischen Wochenschrift erschienen. Ob ich damit die Deutschen so gut suggerieren werde, wie mit dem Hypnotismus bleibt mir vorderhand allerdings sehr zweifelhaft. 
Ich freue mich sehr auf Ihre Rückkehr und wünsche Ihnen einen gelinden Verlauf Ihres Martyriums. Es möge dasselbe gute Früchte tragen.

Mit den herzlichsten Grüssen von uns allen

Ihr Forel

Ihren Bruder sah ich heute in der Klinik.

Wenn ich Ihnen sonst noch dienen kann, verfügen Sie über mich.

Bereits vier Tage später bedankte sich Carl Hauptmann mit dem folgenden Brief, worauf wieder zwei Tage später Forels Antwort erfolgte. Die beiden Briefe lauten:

\section{Carl Hauptmann an August Forel, 4.Juli 1888}

Hochverehrter Herr Professor.

Döbeln 4.7.88.

Für Ihre beiden freundlichen Briefe herzlichsten Dank. Sie haben mir vorzügliche Dienste getan, und vielleicht sogar auch der Sache ein wenig genutzt; denn es gibt doch manchen, dem halb unbewusst innerlich davor graut, wie hohl man wird, je voller man des Alcohols wird, und bei dem ein Körnlein Rates auf guten Boden fällt. Dass die Herren Kameraden meine Abstinenz missliebig bemerkt haben, ist ja allzuleicht verständlich. Ich bin ihnen unheimlich, ich bin in allen Situationen zu nüchtern und gelassen. Selbst nüchtern wissen sie nicht mich zu deuten, und um uns im Kot zu finden, wo man sich nach Heine bekanntlich gleich versteht, dazu geb ich zu wenig Gelegenheit. Wenn Abstinenz auch nur ein Unterscheidungsmerkmal wäre gegen die faden Sippen, gegen die ideal-, pflicht- und opferlosen Philister dieser Welt mit ausgebranntem Hirn und Herzen, so würde ich mich schon um deswillen freudig dazu bekennen und den Schnaps in jeglicher Form als das Medium der Selbstsucht verabscheuen. Übrigens ist mir der Gedanke in schwelgender Gesellschaft Alcohol zu trinken ungefähr so unangenehm, als mich in ein fauliges Schlammbad stürzen zu müssen. Die Grösse des Übels das durch den Alcohol und seinen uneingeschränkten Gebrauch in die Menschenwelt gebracht ist, ist mir doch allmählig allzu klar bewusst geworden und tritt mir nun allzu erschrecklich täglich leibhaftig vors Angesicht. Dass ich die Gelegenheit hier benutze, um wie Dante die Schrecknisse der Hölle so die Schrecknisse des Alcoholismus zu schildern, werden Sie überzeugt sein.

Es wäre ausgezeichnet, wenn ein freisinniger Abstinenzverein in Zürich zu Stande käme und ich würde natürlich mit Freude daran teilnehmen. Denn ich empfinde augenblicklich mehr denn je, wie sehr es Not tut, die Segnungen der Abstinenz: die vorzügliche Steigerung der Arbeitskraft, die Gleichmässigkeit der Stimmung und das lebhafte Bedürfnis idealer Betätigung recht schnell und nachhaltig in weite Kreise zu tragen.

Mit nochmaligem grösstem Danke und herzlichsten Empfehlungen an Sie und Ihre verehrte Familie und in froher Hoffnung auf ein gesundes Wiedersehen in Zürich

Ihr Ihnen aufrichtig ergebener

Carl Hauptmann 
Lieber Freund!

Ihr Brief ist mir eine Erleichterung. Es kamen mir Skrupeln ich hätte meinen ersten Brief zu schroff abgefasst, und, indem ich Ihnen recht helfen wollte, ich hätte über das Ziel geschossen. Nun werde ich durch Ihre freundlichen Zeilen beruhigt.

Vorgestern besuchte ich Ihren Herrn Bruder um ihn zu fragen wann Sie zurückkehren. Ich hörte es sei erst in ca. 3-4 Wochen - und nun greife ich zur Feder. Ich möchte nämlich Sie bitten ob Sie vielleicht die Güte hätten einem Comité beizutreten, das ich zu präsidieren die Ehre habe. Ein Mitglied (unter uns gesagt ein Schafskopf) hat demissioniert, und ich möchte es nicht gerne durch einen anderen Schafskopf ersetzen, den es mir anhängen möchte.

Uebrigens erschrecken Sie nicht. Es handelt sich nur um circa 3 Sitzungen im Jahr, für welche das Protocoll zu führen wäre. Zudem um das Visieren von Circularen bei welchen Sie so gut wie nichts zu tun hätten.

Es handelt sich nämlich um unseren Hülfsverein für Geisteskranke. Die übrigen Comitémitglieder sind recht tüchtige Leute - und es würde uns Gelegenheit geben zusammenzutreffen.

Was meinen Sie dazu? Hätten Sie diese Freundlichkeit? Es würde uns Gelegenheit geben zusammenzutreffen und unsere Abstinenzpläne gegen diese versoffene Menschheit im stillen weiter auszubrüten und zu schmieden.

Es ist natürlich ein Wohltätigkeitsverein. Doch ist die Arbeit recht gering - alles ist ganz geregelt; nur weil uns der Aktuar gerade fehlt wäre diese Stelle auszufüllen, die aber lediglich in dem Führen der Sitzungsprotocolle besteht. Und diese sind möglichst kurz abzufassen.

Ich kann Sie übrigens nur um eine eventuelle Zusage bitten, da nicht ich, sondern das Comité ernennt.

Ich wünsche Ihnen von Herzen bald aus Ihrer militärischen Prüfung erlöst zu sein und verbleibe Ihr aufrichtig ergebener

A. Forel

Mit herzlichem Gruss

Empfehlungen von meiner Frau der es sehr gut geht.

In diesem Antwortschreiben bat Forel Carl Hauptmann, das Amt eines Protokollführers im Hilfsverein für Geisteskranke zu übernehmen, und bereits am 11. Juli 1888 erklärte sich Carl Hauptmann gerne dazu bereit. Die weitere Korrespondenz ist von geringerem Interesse.

Gerhart Hauptmann wird in diesem Briefwechsel wiederholt, aber nur nebenbei, erwähnt. Gewiß sind auch zwischen ihm und August Forel zumindest kleine schriftliche Mitteilungen ausgetauscht worden - man befand sich ja noch nicht im Zeitalter des Telefons -, jedoch wurden uns solche Schriftstücke bisher nicht zugänglich ${ }^{14}$. 
Wie tiefgreifend Gerhart Hauptmann von August Forel beeinflußt wurde, hat er eindrücklich beschrieben. Daß der Einfluß Forels auf seinen Bruder mindestens ebenso nachhaltig war, mußte man annehmen - und diese Briefe beweisen es nun. Carl Hauptmann wurde durch Forel sogar zum eifrigen Alkoholgegner. Davon freilich wollte sein Bruder nichts wissen: «Gelübde dieser Art abzulegen, wäre mir ebenso vorgekommen, als ob ich mich für das ganze Leben an eine Kette gelegt hätte.» ${ }^{15}$ Aber August Forel besaß die liberale Großzügigkeit, beide Brüder Hauptmann gleich freundlich bei sich zu empfangen.

\section{Anmerkungen und Literatur}

${ }^{1}$ Hauptmann, Gerhart, Das Abenteuer meiner Jugend, Berlin 1959, S. 678 ff.

${ }^{2}$ Guthke, Karl S., Gerhart Hauptmann und die Krankheit (Sonderdruck ohne Angabe von Erscheinungsort und -jahr, ca. 1970).

${ }^{3}$ Herrn Prof. K. E. Rothschuh in Münster/Westfalen danke ich für die freundliche Überlassung interessanter Materialien über Carl Hauptmann.

${ }^{4}$ Stroka, Anna, Carl Hauptmanns Werdegang als Denker und Dichter, Wroclaw 1965.

5 Das war wohl auch der Grund, weshalb sein philosophisch-naturwissenschaftliches Hauptwerk erst später erschien: Carl Hauptmann, Die Metaphysik in der modernen Physiologie. Eine kritische Untersuchung. Dresden 1893.

${ }^{6}$ Dieses Haus an der Freiestraße in Zürich 7 hat dem Bauboom bis heute (März 1981) wunderbarerweise getrotzt. Eine Gedenktafel erinnert an die Brüder Hauptmann.

${ }^{7}$ Die beste Einführung in Leben und Werk von August Forel bietet immer noch seine Autobiographie: Rückblick auf mein Leben (2. Auflage, Zürich 1947). - Forels Porträt ist überdies durch die Schweizer Tausendfranken-Banknote weltweit bekannt geworden!

8 Böhtlingk war auch verwandtschaftlich mit dem Waadtland verbunden und machte sich durch eine umfangreiche Biographie des Waadtländer Patrioten César de la Harpe verdient (Friedr. Cäsar Laharpe, 2 Bde., 1925). Dieses Buch ist heute in der Bearbeitung durch Oscar Forel wieder zugänglich (Neuchâtel 1969).

9 Tank, Kurt Lothar, Gerhart Hauptmann, Hamburg 1959 (rowohlt monographien 27).

10 Forel, August, Briefe / Correspondance (Hrsg. Hans H. Walser), Bern 1968.

11 Ich wurde durch die Besprechung des Buches von Anna Stroka (Anm.4) durch Dr. Kurt Speth in Bruchsal («Carl Hauptmann: Eine polnische Biographie», in «Die Tat», Zürich, 25. Mai 1968) angeregt, nach den hier publizierten Briefen zu fahnden. Herr Archivleiter Alexander Münch in Dresden konnte weiterhelfen. Frau Anna Stroka in Wroclaw (Polen) übermittelte mir freundlicherweise Kopien der Briefe. Ihnen allen sei freundlichst gedankt.

${ }^{12}$ Die vorliegende Arbeit wurde an der Henry E.Sigerist-Tagung am 18. Mai 1969 in Morges (Waadt) und am 16. Juni des gleichen Jahres im Institut von Prof. Heinrich Buess in Basel vorgetragen. Die Publikation verzögerte sich, weil eine größere Arbeit über den Problemkreis August Forel/Gerhart Hauptmann geplant war.

13 Anm. 10, S.209f. 
${ }^{14}$ Frau Barbara Hauptmann in Ronco/Ascona teilte mir freundlicherweise mit, daß sich im Briefarchiv von Gerhart Hauptmann in Berlin-West lediglich ein Brief und eine Karte von August Forel an Gerhart Hauptmann befänden. Ein weiterer Brief befindet sich in Privatbesitz in New York.

\section{Summary}

The brothers Carl and Gerhart Hauptmann (the dramatist) were both deeply impressed by the personality and the work of the Swiss psychiatrist Auguste Forel in Zürich, especially by his investigations on hypnotism and his fight against alcoholism. Letters of Carl Hauptmann and of Auguste Forel during the year 1888 are here published for the first time.

Prof. Dr. med. Hans H. Walser

Medizinhistorisches Institut

Rämistraße 71

CH-8006 Zürich 